\title{
Study of Embolic Cerebral Stroke in Atrial Fibrillation Patients. Severity, Recurrence and Outcome
}

Ashraf M. Mohamed Mostafa ${ }^{1}$, Yaser I. Fathy ${ }^{1}$, Mahmoud K. Ahmed ${ }^{2}$, Khaled H. Afifi ${ }^{3}$, Naglaa S. Abd El hady Hammad ${ }^{1}$

Departments of ${ }^{1}$ Anesthesia, Intensive Care \& Pain Management, ${ }^{2}$ Cardiology and ${ }^{3}$ Neurology, Faculty of Medicine, Menoufia University, Menoufia, Egypt

*Corresponding author: Naglaa Samy Abd El hady Hammad, Mobile: (+20)01066361920, Email: naglasamy.ns@ gmail.com

\begin{abstract}
Background: Patients with AF and concomitant potential cardiac sources of embolism increase the risk of recurrent embolism despite anticoagulation.

Objectives: The aim of this study was to study different cardiac sources of embolism in stroke patients with AF and was to study if the presence of concomitant cardiac sources of embolism in AF stroke patients has impact on stroke severity, recurrence, response to treatment $\&$ outcome.

Patients and methods: The study was a prospective clinical study that was conducted on 60 patients admitted to the Intensive Care Unit (ICU), Menoufia University Hospitals. The patients were divided into 2 groups: Group I: Patients with concomitant cardiac sources of embolism. Group II: Patients without concomitant cardiac sources of embolism.

Results: There was significant difference found between the two groups regarding recurrence $(\mathrm{P}=0.045)$. We found that Elders, diabetics, patients with dyslipidemia, patients with CVD, severe National Institutes of Health Stroke Scale (NIHSS) score patients and big infarction size were significantly associated with poor stroke outcome.

Conclusion: In conclusion, we found that most of the recurrent ischemic strokes were associated with elders, diabetics, patients with dyslipidemia, patients with CVD, severe NIHSS score patients and big infarction size.

Keywords: Stroke, Atrial fibrillation, Recurrence, NIHSS score, Infarction.
\end{abstract}

\section{INTRODUCTION}

Approximately $20 \%$ of ischemic strokes are caused by cardioembolic mechanisms (1). Atrial fibrillation (AF) is the most common cause of embolic sources from the heart, and AF-related strokes constitute about $60 \%$ of all cardioembolic strokes ${ }^{(2)}$.

Stroke patients with AF frequently have concomitant potential cardiac sources of embolism. Many of those potential cardiac sources of embolism can be identified through the echocardiographic studies. Transesophageal echocardiography (TEE) is superior to transthoracic echocardiography (TTE) for the detection of potential cardiac sources of embolism in ischemic stroke ${ }^{(3)}$. Some potential cardiac sources of embolism, which include intracardiac thrombus, cardiac tumors, infective and non-infective endocarditis, spontaneous echo contrast (SEC), and patent foramen ovale, are diagnosed mainly based on TEE evaluation. In addition, TEE is the gold standard for the detection of aortic plaques ${ }^{(4)}$. AF patients with coexisting SEC may have increased frequency of intracardiac thrombus and increased risk of stroke. Stroke patients with AF and concomitant cardiac sources of embolism had more severe stroke.

Studies hypothesized that thrombi from AF with the concomitant potential cardiac sources of embolism are larger and/or more resistant to an endogenous thrombolytic system than those from $\mathrm{AF}$ without concomitant potential cardiac sources of embolism, which may lead to more severe stroke. Therefore, we investigated whether stroke severity, recurrence and outcome is different between patients with concomitant potential cardiac sources of embolism and those without among stroke patients with AF.

\section{PATIENTS AND METHODS}

The study was a prospective clinical study that was conducted on 60 patients admitted to the Intensive Care Unit (ICU), Menoufia University Hospitals.

Inclusion criteria: Any adult patient admitted to ICU with stroke and $\mathrm{AF}$.

Exclusion criteria: Patients with relevant proximal arterial occlusion, lacunar stroke, procedural stroke, TIA (transient ischemic attack) and patients who have any contraindication to TEE.

\section{ABSOLUTE CONTRAINDICATIONS:}

Esophageal spasm, esophageal stricture, esophageal laceration, esophageal perforation and esophageal diverticula.

\section{RELATIVE CONTRAINDICATIONS:}

Large diaphragmatic hernia may significantly hinder TEE imaging because of lack of transducer mucosal approximation. Atlantoaxial disease and severe generalized cervical arthritis: TEE should never be performed if there is any question about stability of cervical spine. Patients who received extensive radiation to the mediastinum; this can cause significant difficulty in probe manipulation within the esophagus and is a relative contraindication if the anatomy of the esophagus is not known. Upper gastrointestinal bleeding, significant dysphagia and odynophagia are also relative contraindications. 


\section{Ethical approval:}

The Local Ethics Committee, Faculty of Medicine, Menoufia University approved this study. A written informed consent had been obtained from each of the participants or their legal surrogates.This work has been carried out in accordance with The Code of Ethics of the World Medical Association (Declaration of Helsinki) for studies involving humans.

On the ICU, patients were subjected to the following:

Full history taking: personal history age, sex also to identify any vascular risk factors as (Hypertension, diabetes, hyperlipidemia, smoking and previous cardiovascular diseases (ischemic heart disease and peripheral vascular disease).

History of recurrence: any past history of stroke or TIA.

Neurological examination: The initial stroke severity was assessed by the NIHSS score at admission. We divide stroke severity into: Mild neurological deficit (NIHSS 0-5) Moderate neurological deficit (NIHSS 6-11), Severe neurological deficit (NIHSS 12-18) and Very severe neurological deficit (NIHSS19-•-).

Medication history: Anticoagulant, antiplatelet, statin, angiotensin converting enzyme inhibitor, angiotensin receptor blockers, beta blockers, digoxin and calcium channel blockers.

Laboratory findings: Hemoglobin, white blood cells, platelets, international normalized ratio, initial blood sugar, total cholesterol, triglyceride, high density lipoprotein, and low density lipoprotein .

Radiological investigations: Computerized tomography on the brain (CT brain): The infarction size was measured and carotid duplex to exclude proximal arterial occlusion.

Electrocardiography (ECG): to assess the presence of AF and if there is any ischemic changes.

Transesophageal echocardiography: TEE was performed under local pharyngeal anesthesia with $10 \%$ topical lidocaine before the examination. Gastric, midesophageal, and basal views are obtained and any concomitant cardiac sources of embolism will be diagnosed.

\section{The patients were divided into 2 groups:}

Group I: patients with concomitant cardiac sources of embolism.

Group II: patients without concomitant cardiac sources of embolism.

The stroke severity (NIHSS score, diameter of infarction in CT brain), recurrence, response to thrombolytic therapy will be compared between the 2 groups.

Outcome of stroke: We followed patients after 3 months of enrollment using (mRS modified Rankin scale) and assess recurrence, death, degree of disability.
The Modified Rankin Scale (mRS): The scale runs from 0-6, running from perfect health without symptoms to death.

- No symptoms.

- No significant disability. Able to carry out all usual activities, despite some symptoms.

- Slight disability. Able to look after own affairs without assistance, but unable to carry out all previous activities.

- Moderate disability. Requires some help, but able to walk unassisted.

- Moderately severe disability. Unable to attend to own bodily needs without assistance, and unable to walk unassisted.

- Severe disability. Requires constant nursing care and attention, bedridden, incontinent.

- Dead.

\section{Statistical analysis}

All data were collected, tabulated and statistically analyzed using SPSS 24.0 for windows (SPSS Inc., Chicago, IL, USA). Data were tested for normal distribution using the Shapiro Walk test. Qualitative data were represented as frequencies and relative percentages. Chi-square test $\left(\chi^{2}\right)$ and Fisher exact test were used to calculate difference between qualitative variables as indicated. Quantitative data were expressed as mean $\pm \mathrm{SD}$ (Standard deviation) for parametric and median and range for non-parametric data. All statistical comparisons were two tailed with significance level of $\mathrm{P}$-value $\leq 0.05$ indicates significant, $\mathrm{p}<0.001$ indicates highly significant difference while, $\mathrm{P}>0.05$ indicates Non-significant difference.

\section{RESULTS}

- Table (1) showed that mechanical prosthetic valve was the most concomitant potential cardiac source of embolism and both congestive heart failure and patent foramen ovale were the least sources.

- Table (2) showed that group I had more severe cases $(36.8 \%)$ than group II $(9.1 \%)$ but with no significant difference between the studied groups.

- Table (3) showed that infarction size was bigger in group I compared to group II with statistically significant difference $(\mathrm{p}=0.009)$.

- Table (4) showed that there was significant difference found between the two groups regarding modified Rankin Scale $(\mathrm{P}=0.011)$.

- Table (5) showed that there was significant difference found between the two groups regarding recurrence $(\mathrm{P}=0.045)$.

- Table (6) showed that elders, diabetics, patients with dyslipidemia, patients with CVD, severe NIHSS score patients and big infarction size were significantly associated with poor stroke outcome. 
Table (1): Concomitant potential cardiac sources of embolism among group I

\begin{tabular}{|c|c|c|}
\hline \multirow{2}{*}{ High-risk potential cardiac sources } \\
\cline { 2 - 3 } & \multicolumn{2}{|c|}{$\begin{array}{c}\text { Group I } \\
(\mathbf{n = 3 8})\end{array}$} \\
\hline \multicolumn{2}{|c|}{$\mathrm{N}$} \\
\hline Left atrial thrombus & 8 & 21.1 \\
$>$ Appendage & 3 & 7.9 \\
$>$ Left atrial & 5 & 13.2 \\
\hline Degenerative Mitral stenosis & 8 & 21.1 \\
$>$ Mild & 1 & 2.7 \\
$>$ Moderate & 3 & 7.9 \\
$>$ Severe & 4 & 10.5 \\
\hline Mechanical prosthetic valve & 18 & 47.4 \\
\hline Dilated cardiomyopathy & 10 & 26.3 \\
\hline \multicolumn{1}{|c|}{ Medium-risk potential cardiac sources } \\
\hline Spontaneous echo contrast & 16 & 42.1 \\
\hline Congestive heart failure & 2 & 5.3 \\
\hline Patent foramen ovale & 2 & 5.3 \\
\hline Mitral valve prolapse & 4 & 10.5 \\
\hline Degenerative mitral regurge & 8 & 21.1 \\
$>$ Mild & 2 & 5.3 \\
$>$ Moderate & 3 & 7.9 \\
$>$ Severe & 3 & 7.9 \\
\hline Rheumatic Mitral regurge & 3 & 7.9 \\
\hline
\end{tabular}

Table (2): NIHSS score distribution between the two studied groups

\begin{tabular}{|c|c|c|c|c|c|c|}
\hline \multirow[t]{2}{*}{ Variables } & \multicolumn{2}{|c|}{$\begin{array}{l}\text { Group I } \\
(\mathbf{n}=\mathbf{3 8})\end{array}$} & \multicolumn{2}{|c|}{$\begin{array}{c}\text { Group II } \\
(\mathrm{n}=\mathbf{2 2})\end{array}$} & \multirow[t]{2}{*}{$\chi^{2}$} & \multirow[t]{2}{*}{$\mathbf{P}$} \\
\hline & $\mathbf{N}$ & $\%$ & $\mathbf{N}$ & $\%$ & & \\
\hline Mild & 14 & 36.8 & 10 & 45.5 & \multirow{3}{*}{5.81} & \multirow{3}{*}{.055} \\
\hline Moderate & 10 & 26.3 & 10 & 45.5 & & \\
\hline Severe & 14 & 36.8 & 2 & 9.1 & & \\
\hline
\end{tabular}

$\chi^{2}$ : Chi-square test

Table (3): Infarction size on CT brain between the two studied groups

\begin{tabular}{|l|c|c|c|c|}
\hline Variables & $\begin{array}{c}\text { Group I } \\
(\mathbf{n = 3 8})\end{array}$ & $\begin{array}{c}\text { Group II } \\
(\mathbf{n = 2 2})\end{array}$ & MU & P \\
\hline $\begin{array}{l}\text { Infarction size } \\
\text { Mean } \pm \text { SD }\end{array}$ & $9.35 \pm 7.55$ & $4.71 \pm 3.98$ & 218 & $\mathbf{. 0 0 9}$ \\
\hline
\end{tabular}

MU: Mann Whitney.

Table (4): Modified Rankin Scale (mRS) between the two studied groups

\begin{tabular}{|l|c|c|c|c||}
\hline Variables & $\begin{array}{c}\text { Group I } \\
(\mathbf{n = 3 8})\end{array}$ & $\begin{array}{c}\text { Group II } \\
(\mathbf{n = 2 2})\end{array}$ & \multirow{2}{*}{$\chi^{\mathbf{2}}$} & P \\
\hline No significant disability & $2(5.3 \%)$ & $6(27.3 \%)$ & & \\
\cline { 1 - 3 } Slight disability & $10(26.3 \%)$ & $10(45.5 \%)$ & & \\
\cline { 1 - 3 } Moderate disability & $18(47.3 \%)$ & $3(13.7 \%)$ & \multirow{2}{*}{$\mathbf{1 1 . 2}$} & $\mathbf{. 0 1 1}$ \\
\cline { 1 - 3 } Moderate-severe disability & $2(5.3 \%)$ & $1(4.5 \%)$ & & \\
\cline { 1 - 3 } Severe disability & $4(10.5 \%)$ & $1(4.5 \%)$ & & \\
\hline Dead & $2(5.3 \%)$ & $1(4.5 \%)$ & & \\
\hline
\end{tabular}


Table (5): Recurrence distribution between the two studied groups

\begin{tabular}{|l|c|c|c|c|}
\hline Variables & $\begin{array}{c}\text { Group I } \\
(\mathbf{n = 3 8})\end{array}$ & $\begin{array}{c}\text { Group II } \\
(\mathbf{n = 2 2})\end{array}$ & $\chi^{\mathbf{2}}$ & P \\
\hline Recurrence & $28(73.7 \%)$ & $8(36.4 \%)$ & $\mathbf{8 . 0 9}$ & $\mathbf{. 0 4 5}$ \\
\hline
\end{tabular}

Table (6): Multivariate logistic regression analysis to determine the potential factors associated with stroke poor outcome

\begin{tabular}{|l|c|c|c|c|}
\hline & OR & S.E. & Sig. & 95\% Confidence Interval for OR \\
\hline Age & 1.063 & .152 & $\mathbf{. 0 0 2}$ & $1.021-1.122$ \\
\hline Male gender & 2.264 & .089 & .164 & $.649-4.164$ \\
\hline DM & .913 & .062 & $\mathbf{. 0 0 3}$ & $.716-.943$ \\
\hline HTN & 2.046 & .043 & .466 & $.837-7.232$ \\
\hline Dyslipidemia & 1.423 & .161 & $\mathbf{. 0 1 6}$ & $1.416-1.535$ \\
\hline CVD & 2.233 & .046 & $\mathbf{. 0 4 6}$ & $.495-3.642$ \\
\hline Severe NIHSS & 3.641 & .072 & $\mathbf{. 0 2 1}$ & $1.326-7.161$ \\
\hline Infraction size & 2.014 & .018 & $\mathbf{. 0 3 6}$ & $1.730-2.193$ \\
\hline
\end{tabular}

DM: Diabetes mellitus OR: Odd ratio significance.

HTN: hypertension S.E: standard error

CVD: cardiovascular disease Sig:

\section{DISCUSSION}

$\mathrm{AF}$ represents the most common serious heart rhythm problem and accounts for the majority of all cardioembolic strokes. Importantly, AF-related strokes have been consistently found to portend a poor prognosis. Increasingly, it is understood that AF may relate to the mechanisms of and prognosis from, acute ischemic stroke beyond the known links between $\mathrm{AF}$ and acute cerebral thromboembolism ${ }^{(5)}$. This is an important consideration because AF-patients remain at substantial risk for cerebral infarction even when appropriately treated with oral anticoagulants and the current standard of care for secondary stroke prophylaxis ${ }^{(6)}$.

So this study aimed to describe different cardiac sources of embolism in stroke patients and study if the presence of concomitant cardiac sources of embolism in AF stroke patients has impact on stroke severity, recurrence, response to treatment \& outcome.

The current study found that, mechanical prosthetic valve was the most concomitant potential cardiac source of embolism and both congestive heart failure and patent foramen ovale were the least sources. In agreement with several studies, which reported that, prosthetic valve thrombosis is one of the major sources of thrombosis, which can be lifethreatening. Multimodality imaging is necessary for determination of leaflet immobilization, cause of underlying pathology (thrombus versus pannus or both), and whether thrombolytic therapy attempt in the patient would be successful or surgery is needed ${ }^{(7)}$.

Henninger et al. (8) study found a weak association between a stroke severity score of 2 or more and in-hospital mortality. The driving factors in this relationship were congestive heart failure, history of diabetes, and older age. The mechanism of the associated increased risk of mortality is likely multifactorial. Furthermore, among the variables of severity score, advanced age, congestive heart failure, and diabetes have been associated with short-term mortality after a stroke ${ }^{\left({ }^{(9)}\right.}$. In the same line with other studies, which reported that, hypertension together with age are leading risk factors for silent or symptomatic cerebrovascular disease ${ }^{\mathbf{( 1 0 )}}$.

The prevalence of AF increases with age. It has been shown that $5 \%$ of subjects older than 70 years have $\mathrm{AF}$ (the mean age of patients with $\mathrm{AF}$ is 75 years), and about one fourth of acute strokes in very old patients (> 80 years) are also caused by $\mathrm{AF}^{(\mathbf{1 1})}$.

Future embolism is also more frequent in patients with underlying comorbid heart diseases, such as $\mathrm{AF}$ and stenosis of the mitral valve. The risk of stroke is 3 to 4 times higher in the absence of organic heart disease or risk factors (lone atrial fibrillation). On the other hand, AF is associated with hypertensive heart disease, which is the most common cardiogenic source of cerebral embolization in industrialized countries. Similar rates of cardioembolism for paroxysmal and chronic AF have been reported, so that preventive therapy should not be different for patients with paroxysmal $\mathrm{AF}$ and those with chronic $\mathrm{AF}^{(\mathbf{1 2})}$.

In patients without history of transient ischemic attack (TIA) or stroke, AF carries a risk of stroke of $2 \%-4 \%$ per year. Cardiac emboli arising from cardiac chambers are often large and hence especially likely to cause severe stroke, disability and death ${ }^{(\mathbf{1 2})}$.

A number of cardiac conditions are potential sources of embolism, such as dilated myocardiopathy, heart valve disease (mechanical prosthetic valve, mitral rheumatic stenosis, infectious endocarditis, and marantic endocarditis), left ventricular hypertrophy, atrial myxoma and congenital heart diseases (such as patent foramen ovale, atrial septal aneurysm and ventricular septal defects). Acute coronary syndromes are minor causes of cardioembolism. There is an inverse correlation between ejection fraction of the left ventricle and the incidence of ischemic stroke ${ }^{(\mathbf{1 3})}$. 
In this study, the group with concomitant source had lower mean of HDL level with statistical significant difference $(P=0.041)$. In general, the risk of ischemic stroke in both genders is clearly related with dyslipidemia. In men, low HDL levels are a risk factor for cerebral ischemia but data in women are inconclusive. Because high levels of LDL are clearly related with a higher cardiovascular risk, adequate control of LDL cholesterol is recommended (e.g. National Cholesterol Education Program III guidelines) in subjects without history of cerebrovascular accident ${ }^{(\mathbf{1 4})}$.

In the present study, recurrence was significantly higher among group with concomitant source than other group.

Our study regression analysis found that, elders, diabetics, patients with dyslipidemia, patients with CVD, severe NIHSS score patients and big infarction size were significantly associated with poor stroke outcome. In another studies, age is a continuous risk factor for the occurrence of stroke and dementia, with a two-fold increase in the incidence and prevalence rates for each successive 5 years after age 65 years. On the other hand, men show a higher incidence of cerebral vascular disease than women ${ }^{(15)}$. This is in agreement with Henninger et al. ${ }^{\left({ }^{(8)}\right.}$ study which found that, $\mathrm{AF}$, infarct volume $(\mathrm{p}=0.017)$, pre-admission mRS ( $p<0.001)$, and admission NIHSS ( $<$ 0.001) were independently associated with an unfavorable 90days outcome. However, since previous analyses were not adjusted for potential confounders, it remained to be shown whether AF truly represents an independent risk factor for an unfavorable outcome after CES. In particular, AF is strongly associated with advancing age and other vascular comorbidities, which represent well-recognized risk factors for a poor post-stroke outcome ${ }^{(14)}$.

Henninger et $\boldsymbol{a l} .{ }^{(8)}$ demonstrated that the association between AF and unfavorable outcome was independent of these as well as other critical determinants of the outcome after acute ischemic stroke namely; age, sex, infarct volume, baseline functional deficit, initial deficit severity, and AFrelated vascular comorbidities.

The National Institutes of Health Stroke Scale (NIHSS) provides a reliable, quantitative measure of ischemic stroke severity and is predicted by the infarct size. There was a significant correlation between the infarct volume and NIHSS $(r=0.591 ; \mathrm{P}<0.001)$. This correlation significantly attenuated with increasing leukoaraiosis severity from $\mathrm{r}=0.786(\mathrm{P}<0.001$; absent leukoaraiosis) to $\mathrm{r}=0.498$ ( $\mathrm{P}<0.001$; severe leukoaraiosis) and as shown by ANCOVA ( $<0.001)$. Leukoaraiosis (coefficient, 0.107; 95\% confidence interval, $0.036-0.179 ; \mathrm{P}=0.016$ ) and infarct volume (coefficient, 0.360; 95\% confidence interval, 0.3050.416 ; $\mathrm{P}<0.001$ ) were independently associated with a greater NIHSS deficit in the fully adjusted multivariable model ${ }^{(\mathbf{1 5})}$.
In another study, a multivariate logistic regression model adjusted for sex and race was used to determine the odds ratio (OR) and 95\% confidence interval (CI) for the association between stroke outcome and mortality. The factors associated with increased in-hospital mortality were congestive heart failure (OR, 1.61; 95\% CI, 1.53-1.70), age 75 years or older (OR, 1.27; 95\% CI, 1.19-1.35), and diabetes (OR, 1.24; 95\% CI, 1.14-1.35) ${ }^{(5)}$.

These results strengthen the hypothesis that the known link between AF and poor outcome after stroke involve events not directly related to known AFrelated comorbidities or the embolic event per se, an observation that may serve as the impetus to further investigate potential underlying pathomechanisms ${ }^{(\mathbf{8})}$.

The present study has several strengths and limitations. Strengths relate to (1) inclusion of consecutive patients with imaging confirmed, ischemic, supratentorial, strokes that were evaluated by neurologist using NIHSS score and TEE done by cardiologist; (2) information on stroke severity and (3) adjustment for clinically relevant confounders.

Limitations of our study were related to the relatively small sample size and that we did not quantify leukoaraiosis burden, we did not use CHADS2 VASc in our analysis, and lastly cerebral angiography can't be done.

\section{CONCLUSION}

In conclusion, we found that most of the recurrent ischemic strokes were associated with elders, diabetics, patients with dyslipidemia, patients with CVD, severe NIHSS score patients and big infarction size. This finding emphasizes the need for a more extensive search for specific sources of embolism in individual patients or, alternatively, the establishment of another unifying strategy for antithrombotic therapy addressing different pathways of embolus formation.

\section{REFERENCES}

1. Kimura K, Minematsu K, Yamaguchi T (2005): Atrial fibrillation as a predictive factor for severe stroke and early death in 15,831 patients with acute ischaemic stroke. J Neurol Neurosurg Psychiatry, 76 (5): 679-83.

2. Albers G, Amarenco P, Easton J (2004): Antithrombotic and thrombolytic therapy for ischemic stroke: the Seventh ACCP Conference on Antithrombotic and Thrombolytic Therapy. Chest, 126 (3): 483-512.

3. Kim J, Yoo S, Kwon J (2006): Subtyping of ischemic stroke based on vascular imaging: analysis of 1,167 acute, consecutive patients. J Clin Neurol., 2 (4): 225 30.

4. Han S, Nam H, Kim S (2007): Frequency and significance of cardiac sources of embolism in the TOAST classification. Cerebrovasc Dis., 24 (5): 463-8.

5. Yaghi S (2015): The CHADS2 components are associated with stroke-related in-hospital mortality in patients with atrial fibrillation. Journal of Stroke and Cerebrovascular Diseases, 24 (10): 2404-2407. 
6. Grant P (2007): Diabetes mellitus as a prothrombotic condition'. Journal of Internal Medicine, 262 (2): 157172.

7. Hermans H, Vanassche T, Herijgers $P$ et al. (2013): Antithrombotic therapy in patients with heart valve prostheses. Cardiol Rev., 21(1): 27-36.

8. Henninger $\mathbf{N}$, Goddeau $\mathbf{R}$, Karmarkar A et al. (2016): Atrial fibrillation is associated with a worse 90day outcome than other cardioembolic stroke subtypes. Stroke, 47: 1486-1492.

9. Mancia G (2011): Prognostic value of long-term blood pressure variability: the evidence is growing. Hypertension, 57 (2): 141-3.

10. Mancia G, Bombelli M, Facchetti R et al. (20017): Long-term prognostic value of blood pressure variability in the general population: results of the Pressioni Arteriose Monitorate e Loro Associazioni Study. Hypertension, 49 (6): 1265-70.

11. Arboix A, Font A, Garro C et al. (2007): Recurrent lacunar infarction following a previous lacunar stroke: a clinical study of 122 patients. Journal of Neurology, Neurosurgery, and Psychiatry, 78 (12): 1392-1394.

12. Kishore A, Vail A, Majid A et al. (2014): Detection of atrial fi brillation after ischemic stroke or transient ischemic attack: systematic review and meta-analysis. Stroke, 45: 520-26.

13. Khoo C, Lip G (2009): Clinical outcomes of acute stroke patients with atrial fibrillation', Expert Review of Cardiovascular Therapy. Informa UK Limited, 7 (4): 371-374.

14. Helenius J, Henninger N (2015): Leukoaraiosis Burden Significantly Modulates the Association Between Infarct Volume and National Institutes of Health Stroke Scale in Ischemic Stroke. Stroke, 46 (7): 857-1863.

15. Goldstein L, Adams M, Appel L et al. (2006): Primary prevention of ischemic stroke: a guideline from the American Heart Association.Stroke, 142 (4): 716722. 